\title{
Liver cancer initiation is controlled by AP-1 through SIRT6-dependent inhibition of survivin
}

\author{
Lihua Min, Yuan Ji, Latifa Bakiri, Zhixin Qiu, Jin Cen, Xiaotao Chen, Lingli Chen, Harald Scheuch, Hai Zheng, \\ Lunxiu Qin, Kurt Zatloukal, Lijian Hui and Erwin F. Wagner
}

Nat. Cell Biol. 14, 1203-1211 (2012); published online 7th October 2012; corrected online 14 March 2013

During the preparation of Fig. 8c, the image of c-Fos staining on non-HCC samples was inadvertantly duplicated and presented also as SIRT6 staining of non-HCC samples. The correct Fig. $8 \mathrm{c}$ is shown below.

Furthermore, in the versions of the Article originally published, the sentence starting "This suggests that decreased cell death..." (in the section "Interfering with liver cancer initiation prevents tumorigenesis") was erroneous and should have read: "This suggests that enhanced cell death caused by adenovirus-mediated SIRT6 and SurT34A expression is not maintained beyond the initiation stage." This error has been corrected in the HTML and PDF versions of the Article.

To further clarify Fig. 1a, it should be noted that the $\beta$-actin and c-Jun samples were obtained from separate gels/blots. So, in Supplementary Fig. S9, the uncropped version of the $\beta$-actin blot from Fig. 1a contains three bands on the left from an experiment unrelated to the results shown in Fig 1a.

For further explanation of Figs $1 \mathrm{a}, 2 \mathrm{~b}, 3 \mathrm{~d}, 4 \mathrm{c}$ and $4 \mathrm{~g}$, experimental protein and control $\beta$-actin samples were run on parallel gels and the results were used to derive quantifications. In each case, comparable results have been obtained by assessing experimental protein and $\beta$-actin expression by immunoblotting of samples run on the same gel. In Fig. 5d, experimental protein and SP1 expression were assessed, and quantifications were derived by running samples on parallel gels.

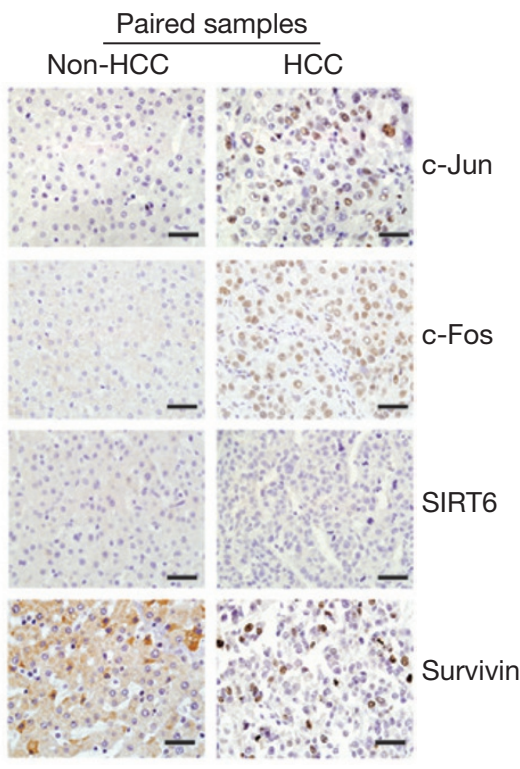

